\title{
NEW RESULTS ON AVERAGING THEORY AND APPLICATIONS
}

\author{
MURILO R. CÂNDIDO AND JAUME LLIBRE
}

\begin{abstract}
The usual averaging theory reduces the computation of some periodic solutions of a system of ordinary differential equations, to find the simple zeros of an associated averaged function. When one of these zeros is not simple, i.e. the Jacobian of the averaged function in it is zero, the classical averaging theory does not provide information about the periodic solution associated to a non simple zero. Here we provide sufficient conditions in order that the averaging theory can be applied also to non simple zeros for studying their associated periodic solutions. Additionally we do two applications of this new result for studying the zero-Hopf bifurcation in the Lorenz system and in the Fitzhugh-Nagumo system.
\end{abstract}

\section{Introduction and Statement of the main Result}

In this paper we introduce an improvement to the first order averaging theorem and use it to study the zero-Hopf bifurcations of periodic orbits which take places in the Lorenz and Fitzhugh-Nagumo systems.

The first order averaging theorem, as presented in [17], can be use to determine periodic orbits coming from the simple zeros of the averaged function, see Theorem 1. Here we use the Lyapunov-Schmidt reduction method(see Lemma 7) in order to make the averaging theorem able to determine periodic solutions coming from degenerated zeros of the averaged function, i.e. zeros for which the Jacobian determinant of the averaged function vanishes, see Theorem 2.

Here a zero-Hopf equilibrium is an equilibrium point of a 3-dimensional autonomous differential system, which has a zero and a pair of pure imaginary eigenvalues. In general the zero-Hopf bifurcation is a 2-parameter unfolding of a 3-dimensional autonomous differential equation with a zeroHopf equilibrium. This kind of zero-Hopf bifurcations has been studied by Guckenheimer, Han, Holmes, Kuznetsov, ... in $[9,8,10,12]$, and it was shown that some complicated invariant sets can bifurcate from the isolated zero-Hopf equilibrium doing the unfolding. Due to the lack of a general theory describing all these kinds of bifurcations that the unfolding of a zeroHopf bifurcation can produce, most of the systems exhibiting this kind of bifurcation must be study directly.

Using Theorem 2, here obtained, we could detect the bifurcation of a periodic orbit from a zero-Hopf bifurcation in the famous Lorenz system of differential equations. As far as we know this was the first time this periodic

2010 Mathematics Subject Classification. Primary: 34C29 37C27.

Key words and phrases. averaging theory, periodic solutions, polynomial differential system, Lorenz system, Fitzhugh-Nagumo system. 
solution was detected. We also apply this theorem to detect the bifurcation of new periodic solutions in the Fitzhugh-Nagumo system, improving the results obtained in [4].

1.1. Averaging theory. The averaging method is a classical theory for studying nonlinear dynamical systems and their periodic solutions. It was conceived by Lagrange in the 18th century, without formal proof, what was only achieved in 1928 by Fatou [5]. After the formalization of the theory important contributions were made by Krylov and Bogoliubov [2] in 1930 and Bogoliubov [1] in 1945. The classical averaging method for computing periodic solutions can be summarized as follows.

We consider the initial value problem

$$
\dot{\mathbf{x}}=\varepsilon \mathbf{F}_{1}(t, \mathbf{x})+\varepsilon^{2} \mathbf{F}_{2}(t, \mathbf{x}, \varepsilon), \quad \mathbf{x}(0)=\mathbf{x}_{0},
$$

and

$$
\dot{\mathbf{y}}=\varepsilon f^{0}(\mathbf{y}), \quad \mathbf{y}(0)=\mathbf{x}_{0},
$$

with $\mathbf{x}, \mathbf{y}$, and $\boldsymbol{x}_{\mathbf{0}}$ in some open $\Omega$ of $\mathbb{R}^{n}, t \in[0, \infty), \varepsilon \in\left[-\varepsilon_{0}, \varepsilon_{0}\right]$. We assume $\mathbf{F}_{1}, \mathbf{F}_{2}$ are $T$-periodic in the variable $t$, and

$$
f^{0}(\mathbf{y})=\frac{1}{T} \int_{0}^{T} \mathbf{F}_{1}(t, \mathbf{y}) d t
$$

Theorem 1. Assume that $\mathbf{F}_{1}, \mathbf{F}_{2}, \mathbf{D}_{\mathbf{x}} \mathbf{F}_{1}, \mathbf{D}_{\mathbf{x x}} \mathbf{F}_{1}$ and $\mathbf{D}_{\mathbf{x}} \mathbf{F}_{2}$ are continuous and bounded by a constant $M$ independent of $\varepsilon$ in $[0, \infty) \times \Omega \times\left[-\varepsilon_{0}, \varepsilon_{0}\right]$, and that $y(t) \in \Omega$ for $t \in[0,1 /|\varepsilon|]$. Then the following statements hold.

(a) For $t \in[0,1 /|\varepsilon|]$ we have $\mathbf{x}(t)-\mathbf{y}(t)=O(\varepsilon)$ as $\varepsilon \rightarrow 0$.

(b) If $\boldsymbol{s}$ is a singular point of system (2) and $\operatorname{det}\left(D_{\mathbf{y}} f^{0}(\boldsymbol{s})\right) \neq 0$, then there exists a T-periodic solution $\Phi(t, \varepsilon)$ of the differential system (1) such that $\Phi(0, \varepsilon) \rightarrow s$ as $\varepsilon \rightarrow 0$.

(c) The stability of the periodic solution $\Phi(t, \varepsilon)$ is given by the stability of the singular point $s$.

For a proof of Theorem 1, see for instance Theorem 11.1, 11.5 and 11.6 of [17], where it is stated for $\varepsilon \in\left[0, \varepsilon_{0}\right)$ but in fact following the proof the same result works for $\varepsilon \in\left[-\varepsilon_{0}, \varepsilon_{0}\right]$, as it is stated here.

The averaged function (3) is a smooth function. Using the notation of Theorem (2), whenever this function vanishes at $\mathcal{Z}$, we have $D f^{0}(z)=$ $0, \forall z \in \mathcal{Z}$. Thus, in order to compute periodic solutions for system (1), we cannot apply Theorem 1 at any singular point $z \in \mathcal{Z}$. Our aim is to show that, under the assumptions of Theorem 2 , is possible to use the LyapunovSchmidt reduction method in order to still apply first order averaging for finding periodic orbits to the differential system (1).

Therefore, using the near-identity relation

$$
\boldsymbol{x}(t)=\mathbf{z}(t)+\varepsilon \boldsymbol{u}(t, \mathbf{z}(t)),
$$

where

$$
\boldsymbol{u}(t, \mathbf{z}(t))=\int_{0}^{t}\left[\mathbf{F}_{1}(s, \mathbf{z})-f^{0}(\mathbf{z})\right] d s,
$$

we do the change of variables $\boldsymbol{x} \rightarrow \mathbf{z}$ and equation (1) becomes

$$
\dot{\mathbf{z}}=\varepsilon f^{0}(\mathbf{z})+\varepsilon^{2} R(t, \mathbf{z})+\mathcal{O}\left(\varepsilon^{3}\right),
$$


where

$$
R(t, \mathbf{z})=\frac{\partial}{\partial \mathbf{z}} \mathbf{F}_{1}(t, \mathbf{z}) \boldsymbol{u}(t, \mathbf{z}(t))-\frac{\partial}{\partial \mathbf{z}} \boldsymbol{u}(t, \mathbf{z}(t)) f^{0}(\mathbf{z})+\mathbf{F}_{2}(t, \mathbf{z}, 0) .
$$

Our main result is:

Theorem 2. Suppose that system (1) satisfies:

(a) $\mathbf{F}_{1}, \mathbf{F}_{2}, \mathbf{D}_{\mathbf{x}} \mathbf{F}_{1}, \mathbf{D}_{\mathbf{x x}} \mathbf{F}_{1}$ and $\mathbf{D}_{\mathbf{x}} \mathbf{F}_{2}$ are continuous and bounded by a constant $M$ independent of $\varepsilon$ in $[0, \infty) \times \Omega$ and $\varepsilon \in[-\bar{\varepsilon}, \bar{\varepsilon}]$.

(b) There exists a $C^{2}$ function $\beta=\left(\beta_{1}, \ldots, \beta_{n-k}\right): \bar{V} \rightarrow \mathbb{R}^{n-k}$ where $V \subset \mathbb{R}^{k}$ is an open and bounded set, such that $f^{0}\left(\mathbf{z}_{\alpha}\right)=0, \forall \mathbf{z}_{\alpha} \in$ $\mathcal{Z}=\{(\alpha, \beta(\alpha)), \alpha \in V\}$.

(c) The Jacobian matrix $D f^{0}\left(\mathbf{z}_{\alpha}\right)$ has in the right up corner the $k \times(n-$ k) matrix $\Gamma_{\alpha}$, while in the right down corner has the $(n-k) \times(n-k)$ matrix $\Delta_{\alpha}$ with $\operatorname{det}\left(\Delta_{\alpha}\right) \neq 0$.

(d) Define the function $h: \bar{V} \rightarrow \mathbb{R}^{k}$ where $h(\alpha)=\int_{0}^{T} R\left(t, \mathbf{z}_{\alpha}\right) d t$. Consider

$$
f^{1}(\alpha)=-\Gamma_{\alpha}\left(\Delta_{\alpha}^{-1} \pi^{\perp} h(\alpha)\right)+\pi h(\alpha)
$$

where $\pi h$ and $\pi^{\perp} h$ are the decomposition of function $h$ in its first $k$ and last $n-k$ coordinates respectively. Assume that there exists $\alpha^{*} \in V$ satisfying the equation $f^{1}\left(\alpha^{*}\right)=0$ such that the Jacobian $\operatorname{det}\left(\left(D f^{1}\right)\left(\alpha^{*}\right)\right) \neq 0$.

Then for $|\varepsilon|$ sufficiently small there exists a T-periodic solution $\Phi(t, \varepsilon)$ of the differential system $(1)$, such that $\phi(0, \varepsilon) \rightarrow\left(\alpha^{*}, \beta\left(\alpha^{*}\right)\right)$ while $\varepsilon \rightarrow 0$.

Theorem 2 is proved in section 2.

Now our objective is to apply Theorems 1 and 2 for studying the periodic orbits bifurcating from zero-Hopf equilibrium points of two important differential systems, the Lorenz and the Fitzhugh-Nagumo.

1.2. Application to Lorenz system. The Lorenz system of differential equations in $\mathbb{R}^{3}$ arose from the work of meteorologist/mathematician Edward N. Lorenz [14], who studied forced dissipative hydro-dynamical systems. It has become one of the most widely studied systems of ODEs because of its wide range of behaviors (see for instance [16]). Although the origins of this system lies in atmospheric modeling, the Lorenz equations also appear in other areas as in the modeling of lasers see [6], and dynamos see [15]. The Lorenz equations are

$$
\begin{aligned}
& \dot{x}=a(x-y), \\
& \dot{y}=x(b-z)-y, \\
& \dot{z}=x y-c z,
\end{aligned}
$$

with $a, b, c$ being real coefficients.

The next result characterizes when the origin of coordinates is a zero-Hopf equilibria of system (7).

Proposition 3. If $a=-1, c=0$ and $b<0$ then there is a 1-parameter family of the Lorenz differential system for which the origin of coordinates is a zero-Hopf equilibrium point. 
Theorem 4. Let $a=-1+a_{2} \varepsilon^{2}$ and $c=c_{1} \varepsilon$. Assume that $b>1, a_{2}<0$, $c_{1} \neq 0$ and $\varepsilon \neq 0$ sufficiently small. Then the Lorenz differential system (7) has a zero-Hopf bifurcation in the equilibrium point at the origin of coordinates, and the following periodic orbit

$$
\begin{aligned}
& x(t, \varepsilon)=2 \varepsilon \sqrt{-2 a_{2}(b-1)} \cos (\sqrt{b-1} t)+\mathcal{O}\left(\varepsilon^{2}\right), \\
& y(t, \varepsilon)=2 \varepsilon \sqrt{-2 a_{2}}(\sin (\sqrt{b-1} t)-\cos (\sqrt{b-1} t))+\mathcal{O}\left(\varepsilon^{2}\right), \\
& z(t, \varepsilon)=-\varepsilon \frac{4 a_{2}(b-1)}{c_{1}}+\mathcal{O}\left(\varepsilon^{2}\right),
\end{aligned}
$$

born at this equilibrium when $\varepsilon=0$.

Theorem 4 is proved using the averaging result given in the new Theorem 2. Proposition 3 and Theorem 4 are proved in section 3 .

1.3. Application to Fitzhugh-Nagumo system. These equations arise in mathematical biology as a model of the transmission of electrical impulses through a nerve axon. The Fitzhugh-Nagumo equations consist in a simplified version of the Hodgkin-Huxley equations which are described using a non-linear diffusion equation coupled to an ordinary differential equation

$$
u_{t}=u_{x x}-f(u)-v, \quad v_{t}=\delta(u-\gamma v),
$$

where $f(u)=u(u-1)(u-a), 0<a<1 / 2$ is a constant, $\delta>0$ and $\gamma>0$ are parameters. In [11] was stated that a single nerve impulse appears to tend, as $t$ increases, to a traveling wave, i.e. a bounded solution $(u, v)(x, t)=(u, v)(\xi)$ where $\xi=x+c t$. Hence one is lead to seek for solutions of $(9)$, not identically zero, of the form $(u, v)=(u(\xi), v(\xi))$ for some $c \neq 0$. Substitution into (9) gives a set of ordinary differential equations which, upon introduction of the variables $x=u, y=v$ and $z=\dot{u}$ takes the form

$$
\begin{aligned}
& \dot{x}=z, \\
& \dot{y}=b(x-d y), \\
& \dot{z}=x(x-1)(x-a)+y+c z,
\end{aligned}
$$

where the dot denotes the derivative with respect to $\xi$ and $(a, b, c, d) \in \mathbb{R}^{4}$ are parameters. For a detailed study concerning traveling waves in (9) see [7]. Hereafter the differential system (10) will be called Fitzhugh-Nagumo differential system.

Proposition 5. There are two parameter families of the Fitzhugh-Nagumo differential systems for which the origin of coordinates is a zero-Hopf equilibrium point, both families are $2-$ parameter. Namely:

(i) for $a d+1=0, b d-c=0$ and $d\left(1-b^{2} d^{3}\right)>0$; and

(ii) for $b=c=0$ and $a<0$.

Proof. A proof for this proposition can be obtained in [4].

Theorem 6. Let $a=-\frac{1}{d}+a_{1} \varepsilon+a_{2} \varepsilon^{2}, c=b d+c_{2} \varepsilon^{2}$ and $\omega=\sqrt{\frac{1-b^{2} d^{3}}{d}}$. Assume that $d\left(1-b^{2} d^{3}\right)>0,(d-1) a_{1} b \neq 0$ and $\varepsilon \neq 0$ sufficiently small. 
Then the Fitzhugh-Nagumo differential system (10) has a zero-Hopf bifurcation in the equilibrium point at the origin of coordinates, and the periodic orbit

$$
\begin{aligned}
x(t, \varepsilon) & =\mathcal{O}\left(\varepsilon^{2}\right), \\
y(t, \varepsilon) & =\mathcal{O}\left(\varepsilon^{2}\right), \\
z(t, \varepsilon) & =\varepsilon \frac{a_{1} d}{d-1}+\mathcal{O}\left(\varepsilon^{2}\right),
\end{aligned}
$$

born at this equilibrium when $\varepsilon=0$.

Moreover if

(a) $b \neq \pm \sqrt{\frac{7 d \pm \sqrt{(d-191)(d+1)}+7}{12 d^{3}(d+1)}}$ and $c_{2} \neq \frac{a_{1}^{2} b d^{3}\left(24 b^{2} d^{3}-13\right)}{24\left(b^{2} d^{3}-1\right)^{2}}$.

Then the Fithugh-Nagumo differential system (10) has four periodic solutions emerging from the origin.

Theorem 6 is proved using Theorem 1 and 2. Theorem 6 is proved in section 4 .

\section{Proof of Theorem 2}

In order to proof Theorem 2 we shall use a particular case of LyapunovSchmidt reduction method for finite dimensional functions. First we need some definitions and notation. In what follows the functions $\pi: \mathbb{R}^{k} \times \mathbb{R}^{n-k} \rightarrow$ $\mathbb{R}^{k}$ and $\pi^{\perp}: \mathbb{R}^{k} \times \mathbb{R}^{n-k} \rightarrow \mathbb{R}^{n-k}$ denote the projections onto the first $k$ coordinates and onto the last $n-k$ coordinates, respectively. We have the following lemma:

Lemma 7. Assume that $k \leq n$ are positive integers. Let $\Omega$ and $V$ be open subsets of $\mathbb{R}^{n}$ and $\mathbb{R}^{k}$, respectively. Let $g_{1}(z)$ and $\beta: \bar{V} \rightarrow \mathbb{R}^{n-k}$ be $\mathcal{C}^{2}$ functions and take $g: \Omega \times(-\varepsilon, \varepsilon) \rightarrow \mathbb{R}^{n}$ such that

$$
g(z, \varepsilon)=g_{0}(z)+\varepsilon g_{1}(z)+\mathcal{O}\left(\varepsilon^{2}\right),
$$

and let $\mathcal{Z}=\left\{z_{\alpha}=(\alpha, \beta(\alpha))\right\} \subset \Omega$. We denote by $\Gamma_{\alpha}$ the upper right corner $k \times(n-k)$ matrix of $D g_{0}\left(z_{\alpha}\right)$, and by $\Delta_{\alpha}$, the lower right corner $(n-k) \times$ $(n-k)$ matrix of $D g_{0}\left(z_{\alpha}\right)$. Assume that for each $z_{\alpha} \in \mathcal{Z}, \operatorname{det}\left(\Delta_{\alpha}\right) \neq 0$ and $g\left(z_{\alpha}\right)=0$. We consider the function $f^{1}: \bar{V} \rightarrow \mathbb{R}^{k}$ as

$$
f^{1}(\alpha)=-\Gamma_{\alpha}\left(\Delta_{\alpha}^{-1} \pi^{\perp} g_{1}\left(z_{\alpha}\right)\right)+\pi g_{1}\left(z_{\alpha}\right) .
$$

If there exists $\alpha^{*} \in V$ with $f^{1}\left(\alpha^{*}\right)=0$ and $\operatorname{det}\left(D f^{1}\left(\alpha^{*}\right)\right) \neq 0$, then there exists $\alpha_{\varepsilon}$ such that $g\left(z_{\alpha_{\varepsilon}}, \varepsilon\right)=0$ and $z_{\alpha_{\varepsilon}} \rightarrow z_{\alpha^{*}}$ when $\varepsilon \rightarrow 0$.

Proof. A proof for this lemma can be found in [13].

Proof of Theorem 2. First we do the change of variables $\mathbf{x} \rightarrow \mathbf{z}$ (4) in order to write the differential system (1) into system (5). The solution $\Phi(t, \mathbf{z}, \varepsilon)$ of equation (5) satisfying $\Phi(0, \mathbf{z}, \varepsilon)=\mathbf{z}$, where $\Phi:[0, \omega) \times \Omega \times\left[-\varepsilon_{0}, \varepsilon_{0}\right] \rightarrow \mathbb{R}^{n}$, is

$$
\Phi(t, \mathbf{z}, \varepsilon)=\mathbf{z}+\varepsilon \int_{0}^{t} f^{0}(\Phi(s, \mathbf{z}, \varepsilon)) d s+\varepsilon^{2} \int_{0}^{t} R(s, \Phi(s, \mathbf{z}, \varepsilon)) d s+\mathcal{O}\left(\varepsilon^{3}\right),
$$


where $\Phi(t, \mathbf{z}, \varepsilon)$ is defined in its maximal interval for positive time $\left[0, \omega_{+}\right) \subset$ $[0, \infty)$. Moreover the solution $\Phi(t, \mathbf{z}, \varepsilon)$ is $T$-periodic if, and only if $\Phi(0, \mathbf{z}, \varepsilon)=$ $\Phi(T, \mathbf{z}, \varepsilon)$, which leads to equation

$$
\begin{aligned}
H(\mathbf{z}, \varepsilon) & =\frac{\Phi(T, \mathbf{z}, \varepsilon)-\mathbf{z}}{\varepsilon} \\
& =\int_{0}^{T} f^{0}(\Phi(s, \mathbf{z}, \varepsilon)) d s+\varepsilon \int_{0}^{T} R(s, \Phi(s, \mathbf{z}, \varepsilon)) d s+\mathcal{O}\left(\varepsilon^{2}\right)=0 .
\end{aligned}
$$

Then we have

$$
\begin{aligned}
\frac{\partial}{\partial \varepsilon} H(\mathbf{z}, \varepsilon) & =\int_{0}^{T} \frac{\partial}{\partial \mathbf{z}} f^{0}(\Phi(s, \mathbf{z}, \varepsilon)) \frac{\partial}{\partial \varepsilon} \Phi(s, \mathbf{z}, \varepsilon) d s+\int_{0}^{T} R(s, \Phi(s, \mathbf{z}, \varepsilon)) d s \\
& +\mathcal{O}(\varepsilon),
\end{aligned}
$$

and from (13) we obtain that

$$
\frac{\partial}{\partial \varepsilon} \Phi(t, \mathbf{z}, \varepsilon)=\int_{0}^{t} f^{0}(\Phi(s, \mathbf{z}, \varepsilon)) d s+\mathcal{O}(\varepsilon) .
$$

Since for $\varepsilon=0$ we have $\Phi(s, \mathbf{z}, 0)=\mathbf{z}$, from (14), (16) and (15) we obtain that

$$
\begin{aligned}
H(\mathbf{z}, 0) & =\int_{0}^{T} f^{0}(\mathbf{z}) d s \\
\frac{\partial}{\partial \varepsilon} \Phi(t, \mathbf{z}, 0) & =\int_{0}^{t} f^{0}(\mathbf{z}) d s \\
\frac{\partial}{\partial \varepsilon} H(\mathbf{z}, 0) & =\int_{0}^{T} \frac{\partial}{\partial \mathbf{z}} f^{0}(\mathbf{z})\left(\int_{0}^{s} f^{0}(\mathbf{z}) d u\right) d s+\int_{0}^{T} R(s, \mathbf{z}) d s,
\end{aligned}
$$

respectively.

Doing the Taylor expansion of the function $H(\mathbf{z}, \varepsilon)$ and using the functions in (17) we obtain that

$$
\begin{aligned}
H(\mathbf{z}, \varepsilon)= & \int_{0}^{T} f^{0}(\mathbf{z}) d s+\varepsilon\left(\int_{0}^{T} \frac{\partial}{\partial \mathbf{z}} f^{0}(\mathbf{z})\left(\int_{0}^{s} f^{0}(\mathbf{z}) d u\right) d s\right. \\
& \left.+\int_{0}^{T} R(s, \mathbf{z}) d s\right)+\mathcal{O}\left(\varepsilon^{2}\right) .
\end{aligned}
$$

Hence the zeros of (18) correspond to periodic solutions of system (5) and provide, going back through the change of variables (4), periodic solutions of system (1).

Notice that function (18) is in the form (12) with

$$
\begin{aligned}
& g_{0}(\mathbf{z})=\int_{0}^{T} f^{0}(\mathbf{z}) d s, \\
& g_{1}(\mathbf{z})=\int_{0}^{T} \frac{\partial}{\partial \mathbf{z}} f^{0}(\mathbf{z})\left(\int_{0}^{s} f^{0}(\mathbf{z}) d u\right) d s+\int_{0}^{T} R(s, \mathbf{z}) d s .
\end{aligned}
$$

Thus the result follows by Lemma 7 . In addition, using the notation of Lemma 7 observe that $g_{1}\left(z_{\alpha}\right)=h(\alpha)$, where $h(\alpha)$ is defined in $(d)$ of Theorem 2.

In this section we prove Proposition 3 and Theorem 4. 


\section{Application to Lorenz system}

Proof of Proposition 3. The Jacobian matrix of system (7) evaluated at the origin is

$$
M_{0}=\left(\begin{array}{ccc}
-a & a & 0 \\
b & -1 & 0 \\
0 & 0 & -c
\end{array}\right) .
$$

Thus its characteristic polynomial is $P(\lambda)=\lambda(a(b-1)-(a+1) c)+a(b-$ 1) $c+\lambda^{2}(-a-c-1)-\lambda^{3}$, consequently the origin is a zero-Hopf equilibrium point if, and only if $P(\lambda)=-\lambda\left(\lambda^{2}+\omega^{2}\right)$ with $\omega>0$. This equation has only one real solution $a=-1, c=0$ and $b>1$ proving the proposition.

Proof of Theorem 4. Assume $a=-1+a_{2} \varepsilon^{2}$ and $c=c_{1} \varepsilon$ in system (7). Doing the change of variables $(x, y, z) \rightarrow \varepsilon(X, Y, Z)$ the system becomes

$$
\begin{aligned}
\dot{X} & =\varepsilon^{2} a_{2}(Y-X)+X-Y, \\
\dot{Y} & =-\varepsilon X Z+b X-Y, \\
\dot{Z} & =\varepsilon\left(X Y-c_{1} Z\right) .
\end{aligned}
$$

In order to write the linear part of system (19) in its normal Jordan form, we do the change of variables $\bar{x}=X, \bar{x}=\sqrt{b-1} Y+X$ and $\bar{z}=Z$ obtaining

$$
\begin{aligned}
& \dot{\bar{x}}=-\sqrt{b-1} \bar{y}+a_{2} \varepsilon^{2} \sqrt{b-1} \bar{y}, \\
& \dot{\bar{y}}=\sqrt{b-1} \bar{x}-\varepsilon \frac{\overline{x z}}{\sqrt{b-1}}-\varepsilon^{2} a_{2} \bar{y}, \\
& \dot{\bar{z}}=\varepsilon\left(\sqrt{b-1} \bar{x} \bar{y}-c_{1} \bar{z}+\bar{x}^{2}\right) .
\end{aligned}
$$

Using the cylindrical coordinates $\bar{x}=r \cos \theta, \bar{y}=r \sin \theta$ and $\bar{z}=z$ in system (20) we have

$$
\begin{aligned}
& \dot{r}=-\varepsilon \frac{r z \sin \theta \cos \theta}{\sqrt{b-1}}+\varepsilon^{2} a_{2} r \sin \theta(\sqrt{b-1} \cos \theta-\sin \theta), \\
& \dot{\theta}=\sqrt{b-1}-\varepsilon \frac{z \cos ^{2} \theta}{\sqrt{b-1}}-\varepsilon^{2} a_{2} \sin \theta(\sqrt{b-1} \sin \theta+\cos \theta), \\
& \dot{z}=\varepsilon\left(r^{2} \cos \theta(\sqrt{b-1} \sin \theta+\cos \theta)-c_{1} z\right) .
\end{aligned}
$$

Taking $\theta$ as the new independent variable in system (21) we came to system

$$
\begin{aligned}
\frac{d r}{d \theta}= & -\varepsilon \frac{r z \sin \theta \cos \theta}{b-1}+\varepsilon^{2} r \sin \theta\left(-\frac{a_{2} \sin \theta}{\sqrt{b-1}}+a_{2} \cos \theta-\frac{z^{2} \cos ^{3} \theta}{(b-1)^{2}}\right) \\
& +\varepsilon^{3} \mathbf{F}_{31}(\theta,(r, z), \varepsilon), \\
\frac{d z}{d \theta}= & \varepsilon \frac{\left(r^{2}(\sqrt{b-1} \sin (2 \theta)+\cos (2 \theta))-2 c_{1} z+r^{2}\right)}{2 \sqrt{b-1}} \\
& +\varepsilon^{2} \frac{z \cos ^{2} \theta\left(r^{2}(\sqrt{b-1} \sin (2 \theta)+\cos (2 \theta))-2 c_{1} z+r^{2}\right)}{2(b-1)^{3 / 2}} \\
& +\varepsilon^{3} \mathbf{F}_{32}(\theta,(r, z), \varepsilon) .
\end{aligned}
$$


The system (22) is in the normal form for applying the averaging theorem with $T=2 \pi$. Function (3) corresponding to (22) is

$$
f^{0}(r, z)=\left(0, \frac{r^{2}-2 c_{1} z}{2 \sqrt{b-1}}\right) .
$$

Equation (23) has the continuum of zeros $\mathcal{Z}=\left\{\left(\alpha, \frac{\alpha^{2}}{2 c_{1}}\right): \alpha \in \mathbb{R}\right\}$. Applying Theorem 2, function (6) corresponding to system (22) is

$$
f^{1}(\alpha)=-\frac{\pi \alpha\left(8 a_{2}(b-1)+\alpha^{2}\right)}{8(b-1)^{3 / 2}} .
$$

The positive zero of function $(24)$ is $\alpha^{*}=2 \sqrt{-2 a_{2}(b-1)}$. Furthermore, $D f^{1}\left(\alpha^{*}\right)=\frac{2 a_{2} \pi}{\sqrt{b-1}}$ so by Theorem 2 there is a periodic solution emerging from $\left(\alpha^{*}, \frac{\alpha^{* 2}}{2 c_{1}}\right)$. Thus we can write the following periodic solution for system $(22)$

$$
(r(\theta, \varepsilon), z(\theta, \varepsilon))=\left(2 \sqrt{-2 a_{2}(b-1)},-\frac{4 a_{2}(b-1)}{c_{1}}\right)+\mathcal{O}(\varepsilon),
$$

consequently system (21) has the periodic solution

$$
(r(t, \varepsilon), \theta(t, \varepsilon), z(t, \varepsilon))=\left(2 \sqrt{-2 a_{2}(b-1)}, \sqrt{b-1} t,-\frac{4 a_{2}(b-1)}{c_{1}}\right)+\mathcal{O}(\varepsilon) .
$$

Going back through the cylindrical coordinate change we find the periodic solution $(\bar{x}(t, \varepsilon), \bar{y}(t, \varepsilon), \bar{z}(t, \varepsilon))=$

$$
\begin{aligned}
& \left(2 \sqrt{-2 a_{2}(b-1)} \cos (\sqrt{b-1} t), 2 \sqrt{-2 a_{2}(b-1)} \sin (\sqrt{b-1} t),-\frac{4 a_{2}(b-1)}{c_{1}}\right) \\
& +\mathcal{O}(\varepsilon) .
\end{aligned}
$$

for system (20). Finally undoing the linear change of variables and the rescaling of system (19) we obtain the periodic solution (8) for system (7) under the conditions of Theorem 4.

\section{Application to Fitzhugh-Nagumo system}

The following lemma will be useful in the proof of Theorem 6 , it was proved in [3].

Lemma 8. Consider $p+1$ linearly independent functions $\xi_{i}: I \subset \mathbb{R} \rightarrow \mathbb{R}$, $i=0,1, \ldots, p$.

(a) Given $p$ arbitrary values $x_{i} \in I, i=1,2, \ldots, p$ there exist $p+1$ constants $C_{i}, i=0,1, \ldots, p$ such that

$$
\xi(x):=\sum_{i=0}^{p} C_{i} \xi_{i}(x)
$$

is not the zero function and $\xi\left(x_{i}\right)=0$ for $i=1,2, \ldots, p$.

(b) Furthermore, if all $\xi_{i}$ are analytical functions on $I$ and there exists $j \in 1,2, \ldots, p$ such that $\xi_{j} \Upsilon_{I}$ has a constant sign, it is possible to get an $\xi$ by (25), such that it has at least $p$ simple zeroes in $I$. 
Proof of Theorem 6. Assuming $a=-\frac{1}{d}+a_{1} \varepsilon+a_{2} \varepsilon^{2}, c=b d+c_{2} \varepsilon^{2}$ and $d\left(1-b^{2} d^{3}\right)>0$. Doing the change of variables $(x, y, z) \rightarrow \varepsilon(X, Y, Z)$ system (10) becomes

$$
\begin{aligned}
\dot{X}= & Z, \\
\dot{Y}= & b(X-d Y), \\
\dot{Z}= & -\frac{X}{d}+Y+d b Z+\varepsilon \frac{a_{1} d+X(1-d)}{d} \\
& +\varepsilon^{2}\left(X\left(a_{2}+X\left(X-a_{1}\right)\right)+c_{2} Z\right)-a_{2} X^{2} \varepsilon^{3},
\end{aligned}
$$

in order to write the linear part of system (26) in its normal Jordan form we do the following change of variables $X=\bar{x}+\frac{b d}{\omega} \bar{y}+\bar{z}, Y=\frac{b}{\omega} \bar{y}+\frac{\bar{z}}{d}$, $Z=b d \bar{x}-\omega \bar{y}$ with $\omega=\sqrt{\frac{1}{d}-b^{2} d^{2}}$, obtaining

$$
\begin{aligned}
\dot{\bar{x}}= & -\omega \bar{y} \\
\dot{\bar{y}}= & \omega \bar{x}+\frac{\varepsilon}{d \omega^{3}}((b d \bar{y}+(\bar{x}+\bar{z}) \omega))\left(b(d-1) d \bar{y}-\left(a_{1} d-(d-1)(\bar{x}+\bar{z})\right) \omega\right) \\
& -\frac{\varepsilon^{2}}{\omega}\left(\left(a_{2}+\left(\bar{x}+\bar{z}+\frac{b d \bar{y}}{\omega}\right)\left(-a_{1}+\bar{x}+\bar{z}+\frac{b d \bar{y}}{\omega}\right)\right)\left(\bar{x}+\bar{z}+\frac{b d \bar{y}}{\omega}\right)\right. \\
& \left.+c_{2}(b d \bar{x}-\omega \bar{y})\right), \\
\dot{\bar{z}}= & -\frac{\varepsilon b}{\omega^{4}}(b d \bar{y}+\omega(\bar{x}+\bar{z}))\left(b(d-1) d y-\omega\left(a_{1} d-(d-1)(\bar{x}+\bar{z})\right)\right) \\
& +\varepsilon^{2} \frac{b d}{\omega^{2}}\left(( \frac { b d \overline { y } } { \omega } + \overline { x } + \overline { z } ) \left(\left(\frac{b d \bar{y}}{\omega}+\bar{x}+\bar{z}\right)\left(\frac{b d \bar{y}}{\omega}+\bar{x}+\bar{z}-a_{1}\right)\right.\right. \\
& \left.\left.+a_{2}\right)+c_{2}(b d \bar{x}-\bar{y} \omega)\right)+\mathcal{O}\left(\varepsilon^{3}\right),
\end{aligned}
$$

Using the change of variables $(\bar{x}, \bar{y}, \bar{z}) \rightarrow(u, v, w)$ where $\bar{x}=u \cos (\omega t)-$ $v \sin (\omega t), \bar{y}=v \cos (\omega t)+u \sin (\omega t)$ and $\bar{z}=w$, system (27) becomes

$$
\begin{aligned}
\dot{u}= & \frac{\varepsilon \sin (t \omega)}{d \omega^{3}}(\sin (t \omega)(b d u-v \omega)+\cos (t \omega)(b d v+u \omega) \\
& +\omega w)((d-1)(\sin (t \omega)(b d u-v \omega)+\cos (t \omega)(b d v+u \omega)) \\
& \left.-\omega\left(a_{1} d-d w+w\right)\right)-\frac{\varepsilon^{2} \sin (t \omega)}{\omega}\left(\left(\sin (t \omega)\left(\frac{b d u}{\omega}-v\right)\right.\right. \\
& \left.+\cos (t \omega)\left(\frac{b d v}{\omega}+u\right)+w\right)\left(\frac{1}{\omega^{2}}((\sin (t \omega)(b d u-v \omega)+\cos (t \omega)(b d v\right. \\
& \left.+u \omega)+\omega w)\left(\omega\left(w-a_{1}\right)+\sin (t \omega)(b d u-v \omega)+\cos (t \omega)(b d v+u \omega)\right)\right) \\
& \left.\left.+a_{2}\right)-c_{2}(\sin (t \omega)(b d v+u \omega)+\cos (t \omega)(v \omega-b d u))\right)+\mathcal{O}\left(\varepsilon^{3}\right), \\
\dot{v}= & \frac{\varepsilon \cos (t \omega)}{d \omega^{3}}(\sin (t \omega)(b d u-v \omega)+\cos (t \omega)(b d v+u \omega)+\omega w)((d \\
& \left.-1)(\sin (t \omega)(b d u-v \omega)+\cos (t \omega)(b d v+u \omega))-\omega\left(a_{1} d-d w+w\right)\right)
\end{aligned}
$$




$$
\begin{aligned}
& -\frac{\varepsilon^{2} \cos (t \omega)}{\omega}\left(\left(\sin (t \omega)\left(\frac{b d u}{\omega}-v\right)+\cos (t \omega)\left(\frac{b d v}{\omega}+u\right)+w\right)\right. \\
& \left(\frac { 1 } { \omega ^ { 2 } } ( \operatorname { s i n } ( t \omega ) ( b d u - v \omega ) + \operatorname { c o s } ( t \omega ) ( b d v + u \omega ) + \omega w ) \left(\omega\left(w-a_{1}\right)\right.\right. \\
& \left.+\sin (t \omega)(b d u-v \omega)+\cos (t \omega)(b d v+u \omega))+a_{2}\right)-c_{2}(\sin (t \omega)(b d v \\
& +u \omega)+\cos (t \omega)(v \omega-b d u)))+\mathcal{O}\left(\varepsilon^{3}\right) \\
\dot{w}= & \frac{\varepsilon b}{\omega^{4}}(\sin (t \omega)(b d u-v \omega)+\cos (t \omega)(b d v+u \omega)+\omega w)\left(\omega\left(a_{1} d-d w+w\right)\right. \\
& -(d-1)(\sin (t \omega)(b d u-v \omega)+\cos (t \omega)(b d v+u \omega))) \\
& +\frac{\varepsilon^{2} b d}{\omega^{2}}\left(\left(\sin (t \omega)\left(\frac{b d u}{\omega}-v\right)+\cos (t \omega)\left(\frac{b d v}{\omega}+u\right)+w\right)\right. \\
& \left(\frac { 1 } { \omega ^ { 2 } } ( \operatorname { s i n } ( t \omega ) ( b d u - v \omega ) + \operatorname { c o s } ( t \omega ) ( b d v + u \omega ) + \omega w ) \left(\omega\left(w-a_{1}\right)\right.\right. \\
& \left.+\sin (t \omega)(b d u-v \omega)+\cos (t \omega)(b d v+u \omega))+a_{2}\right)-c_{2}(\sin (t \omega)(b d v+u \omega) \\
& +\cos (t \omega)(v \omega-b d u)))+\mathcal{O}\left(\varepsilon^{3}\right) .
\end{aligned}
$$

The above system is under the normal form for applying the averaging Theorems 1 and 2 , where $T=\frac{2 \pi}{\omega}, \mathbf{y}=(u, v, w)$ and the function (2) corresponding to system $(28)$ is

$$
\begin{aligned}
f^{0}(\mathbf{y})= & -\left(\frac{\left(a_{1} d-2(d-1) w\right)(b d u-v \omega)}{2 d \omega^{2}}, \frac{\left(a_{1} d-2(d-1) w\right)(b d v+u \omega)}{2 d \omega^{2}}\right. \\
& \left.\frac{b\left((d-1)\left(u^{2}+v^{2}\right)\left(b^{2} d^{2}+\omega^{2}\right)-2 \omega^{2} w\left(a_{1} d-d w+w\right)\right)}{2 \omega^{4}}\right) .
\end{aligned}
$$

The zeros of this function are $\mathbf{s}=\left(0,0, \frac{a_{1} d}{d-1}\right)$ and the continuum of zeros $\mathcal{Z}_{ \pm}=\left\{\left(\alpha, \beta_{ \pm}(\alpha)\right), \alpha \in \mathbb{R}\right\}$ where

$$
\beta_{ \pm}(\alpha)=\left( \pm \sqrt{\frac{\omega^{2}\left(a_{1}^{2} d^{2}-2(d-1)^{2} \alpha^{2}\right)-2 b^{2}(d-1)^{2} d^{2} \alpha^{2}}{2(d-1)^{2}\left(b^{2} d^{2}+\omega^{2}\right)}}, \frac{a_{1} d}{2(d-1)}\right)
$$

Furthermore, since $\operatorname{det}\left(D f^{0}(\mathbf{s})\right)=-\frac{a_{1}^{3} b d\left(b^{2} d^{2}+\omega^{2}\right)}{4 \omega^{6}} \neq 0$ by Theorem 1 we can ensure the existence of a periodic solution of system (28) emerging from the origin. Consequently, going back through the change of variables, system (10) has the periodic solution (11).

For $\mathbf{z}_{\alpha} \in \mathcal{Z}_{-}$, we have

$$
f^{1}(\alpha)=\frac{\pi a_{1}^{2} d^{2} \sqrt{b^{2} d^{2}+\omega^{2}}\left(a_{1}^{2} b d+24 c_{2} \omega^{4}\right)}{24(d-1) \omega^{4}\left(2(d-1) \alpha \omega \sqrt{b^{2} d^{2}+\omega^{2}}-b d \Lambda_{\alpha}\right)},
$$


where $\Lambda_{\alpha}=\sqrt{a_{1}^{2}\left(2 d-2 b^{2} d^{4}\right)-\frac{4(d-1)^{2} \alpha^{2}}{d}}$. As $f^{1}(\alpha) \neq 0 \forall \alpha \in \mathbb{R}$ we cannot apply Theorem 2 in this case. On the other hand, for $\mathbf{z}_{\alpha} \in \mathcal{Z}_{+}$,

$$
f^{1}(\alpha)=\frac{C_{0}+C_{1} \alpha^{2}+C_{2} \alpha^{4}+C_{3} \Lambda_{\alpha} \alpha+C_{4} \Lambda_{\alpha} \alpha^{3}}{24(d-1)^{2} d \omega^{8}\left(2(d-1) \alpha \omega \sqrt{b^{2} d^{2}+\omega^{2}}-b d \Lambda_{\alpha}\right)},
$$

where

$$
\begin{aligned}
C_{0}= & \pi\left(-a_{1}^{2}\right)(d-1) d^{3} \omega^{4}\left(b^{2} d^{2}+\omega^{2}\right)\left(a_{1}^{2} b d+24 c_{2} \omega^{4}\right), \\
C_{1}= & 4 \pi(d-1)^{3} d \omega^{2}\left(b^{2} d^{2}+\omega^{2}\right)\left(a_{1}^{2}\left(13 b^{3} d^{3}-11 b d \omega^{2}\right)+24 c_{2} \omega^{4}\left(b^{2} d^{2}+\omega^{2}\right)\right), \\
C_{2}= & -96 \pi b(d-1)^{5}(b d-\omega)(b d+\omega)\left(b^{2} d^{2}+\omega^{2}\right)^{2} \\
C_{3}= & 2 \pi d \omega^{3} \sqrt{b^{2} d^{2}+\omega^{2}}\left(a _ { 1 } ^ { 2 } \left(b^{2} d^{2}\left(3 d(d+8) \omega^{2}-14(d-1)^{2}\right)+3 d(d+8) \omega^{4}\right.\right. \\
& \left.\left.-2(d-1)^{2} \omega^{2}\right)+24 a_{2}(d-1)^{2} \omega^{2}\left(b^{2} d^{2}+\omega^{2}\right)\right) \\
C_{4}= & 96 \pi b^{2}(d-1)^{4} d \omega\left(b^{2} d^{2}+\omega^{2}\right)^{3 / 2}
\end{aligned}
$$

The denominator of $f^{1}$ vanishes at $\alpha^{ \pm}= \pm \sqrt{\frac{a_{1}^{2} b^{2} d^{5}\left(1-b^{2} d^{3}\right)}{2(d-1)^{2}}}$. In addition, the domain of definition of $\Lambda_{\alpha}$ is the interval $J \subset \mathbb{R}$ such that $|\alpha| \leq\left|\frac{d}{(d-1)}\right| \sqrt{\frac{a_{1}^{2} d\left(1-b^{2} d^{3}\right)}{2}}$. Thus, the domain of definition of $f^{1}$ is $I=J \backslash\left\{\alpha^{ \pm}\right\}$.

In order to study the maximum number of simple zeros of function (29) we are going to apply Lemma 8 . Thus, we define the functions $\xi_{0}(\alpha)=1$, $\xi_{1}(\alpha)=\alpha^{2}, \xi_{2}(\alpha)=\alpha^{4}, \xi_{3}(\alpha)=\Lambda_{\alpha} \alpha, \xi_{4}(\alpha)=\Lambda_{\alpha} \alpha^{3}$ and $\xi(\alpha)=\sum_{i=0}^{4} C_{i} \xi_{i}(\alpha)$ with $\alpha \in I \subset \mathbb{R}$. We observe that the five functions of the set $\left\{C_{i} \mid i=\right.$ $0,1, \ldots, 4\}$ are linearly independent. Indeed due to hypothesis $(a)$, the determinant of the Jacobian matrix $\frac{\partial\left(C_{0}, C_{1}, C_{2}, C_{3}, C_{4}\right)}{\partial\left(a_{1}, a_{2}, b, c_{2}, d\right)}$ is

$$
\begin{aligned}
& -\frac{42467328 \pi^{5} a_{1} b^{2}(d-1)^{14}\left(b^{2} d^{3}-1\right)^{7}}{d^{14}}\left(d\left(b^{2}(d+1) d^{2}\left(6 b^{2} d^{3}-7\right)+2\right)\right. \\
& +10)\left(a_{1}^{2} b d^{3}\left(24 b^{2} d^{3}-13\right)-24 c_{2}\left(b^{2} d^{3}-1\right)^{2}\right) \neq 0 .
\end{aligned}
$$

Thus statement $(a)$ of Lemma 8 ensures that the function $\xi(\alpha)$ has 4 zeros. In addiction, $\xi_{0}(\alpha)$ does not changes sign, so by statement $(b)$ of the same lemma, $\xi(\alpha)$ has 4 simple zeros. Finally, we observe that the simple zeros of $\xi(\alpha)$ are also simple zeros of $f^{1}(\alpha)$ so there exist values $\alpha_{i} \in I$ for $i=$ $1,2,3,4$ such that $f^{1}\left(\alpha_{i}\right)=0$ and $\operatorname{det}\left(D f^{1}\left(\alpha_{i}\right)\right) \neq 0$. So the conclusion of Theorem 6 follows from Theorem 2 .

\section{ACKNOWLEDGEMENTS}

The first author is supported by CNPq 248501/2013-5. The second author is partially supported by a MINECO grant MTM2013-40998-P, an AGAUR grant number 2014 SGR568, the grants FP7-PEOPLE-2012-IRSES 318999 
and 316338, and a CAPES grant 88881.030454 /2013-01 from the Program CSF-PVE.

\section{REFERENCES}

[1] N. N. Bogoliubov, On some statistical methods in mathematical physics, Izv. Akad. Nauk Ukr. SSR, Kiev, 1945.

[2] N. N. Bogoliubov AND N. KRYLOv, The application of methods of nonlinear mechanics in the theory of stationary oscillations, Publ. 8 of the Ukrainian Acad. Sci. Kiev, 1934.

[3] B. Coll, A. Gasull and R. Prohens, Bifurcation of limit cycles from two families of centers, Dyn. Contin. Discrete Impuls. Syst. 12 (2005), 275-288.

[4] R. D. EuzÉBio, J. Llibre And C. Vidal, Zero-Hopf bifurcation in the FitzHughNagumo system, to appear in Mathematical Methods in Applied Sciences.

[5] P. Fatou, Sur le mouvement d'un systàme soumis à des forces à courte période, Bull. Soc. Math. France 56 (1928), 98-139.

[6] H. HAKEn, Analogy between higher instabilities in fluids and lasers, Physics Letters A 53 (1975), 77-78.

[7] W. GaO And J. WANG, Existence of wavefronts and impulses to FitzHugh-Nagumo equations. Nonlinear Anal. 57 (2004), 667-676.

[8] J. Guckenheimer And P. Holmes, Nonlinear oscillations, dynamical systems, and bifurcations of vector fields, revised and corrected reprint of the 1983 original. Applied Mathematical Sciences 42, Springer-Verlag, New York, 1990.

[9] J. Guckenheimer, On a codimension two bifurcation, Lecture Notes in Mat. 828 (1980), 99-142.

[10] M. HAN, Existence of periodic orbits and invariant tori in codimension two bifurcations of three dimensional system, J. Sys. Sci \& Math. Scis. 18 (1998), 403-709.

[11] S. P. Hastings, Some Mathematical Problems from Neurobiology The American Mathematical Monthly Vol. 82, No. 9 (Nov. 1975), 881-895.

[12] Y. A. Kuznetsov, Elements of Applied Bifurcations Theory, Springer-verlag, 3rd edition, 2004.

[13] J. LliBRE AND D. D. NovAEs, Improving the averaging theory for computing periodic solutions of the differential equations, Zeitschrift für angewandte Mathematik und Physik (2015) 1401-1412.

[14] E.N. Lorenz, Deterministic nonperiodic flow, J. Atmos. Sci. 20 (1963), 130-141.

[15] E. Knobloch, Chaos in the segmented disc dynamo, Physics Letters A 82 (1981), 439-440.

[16] C. Sparrow, The Lorenz Equations: Bifurcations, Chaos and Strange Attractors, Appl. Math. Sci., 41, Springer-Verlag, New Yourk 1982.

[17] F. Verhulst, Nonlinear Differential Equations and Dynamical Systems, Universitext, Springer, 1991.

${ }^{1}$ Departament de Matemàtiques, Universitat Autònoma de Barcelona, 08193 Bellaterra, Barcelona, Catalonia, Spain

E-mail address: candidomr@mat.uab.cat

${ }^{1}$ Departament de Matemàtiques, Universitat Autònoma de Barcelona, 08193

Bellaterra, Barcelona, CAtalonia, Spain

E-mail address: jllibre@mat.uab.cat 